\title{
Multiple Modes of Phase Locking between Sniffing and Whisking during Active Exploration
}

\author{
Sachin Ranade, ${ }^{1 \star}$ Balázs Hangya, ${ }^{1,2 \star}$ and Adam Kepecs ${ }^{1}$ \\ ${ }^{1}$ Cold Spring Harbor Laboratory, Cold Spring Harbor, New York 11724, and ${ }^{2}$ Laboratory of Cerebral Cortex Research, Institute of Experimental Medicine, \\ Hungarian Academy of Sciences, Budapest H-1083, Hungary
}

\begin{abstract}
Sense organs are often actively controlled by motor processes and such active sensing profoundly shapes the timing of sensory information flow. The temporal coordination between different active sensing processes is less well understood but is essential for multisensory integration, coordination between brain regions, and energetically optimal sampling strategies. Here we studied the coordination between sniffing and whisking, the motor processes in rodents that control the acquisition of smell and touch information, respectively. Sniffing, high-frequency respiratory bouts, and whisking, rapid back and forth movements of mystacial whiskers, occur in the same theta frequency range $(4-12 \mathrm{~Hz})$ leading to a hypothesis that these sensorimotor rhythms are phase locked. To test this, we monitored sniffing using a thermocouple in the nasal cavity and whisking with an electromyogram of the mystacial pad in rats engaged in an open field reward foraging behavior. During bouts of exploration, sniffing and whisking showed strong one-to-one phase locking within the theta frequency range $(4-12 \mathrm{~Hz})$. Interestingly, we also observed multimode phase locking with multiple whisks within a sniff cycle or multiple sniffs within a whisk cycle-always at the same preferred phase. This specific phase relationship coupled the acquisition phases of the two sensorimotor rhythms, inhalation and whisker protraction. Our results suggest that sniffing and whisking may be under the control of interdependent rhythm generators that dynamically coordinate active acquisition of olfactory and somatosensory information.
\end{abstract}

\section{Introduction}

Behavioral strategies to acquire information about the environment can be divided into two broad classes: "sensing-to-act" or discrimination and "acting-to-sense" or exploration. Active sensing is common to many phyla in the animal kingdom across different sensory modalities, from head casting in Drosophila larvae (Gomez-Marin et al., 2011), to antennal flicking in crustaceans (Atema, 1995) and saccadic eye movements in humans (Otero-Millan et al., 2008). Rodents rhythmically sample the olfactory world around them with short bouts of rapid respiration termed sniffing (Welker, 1964; Youngentob et al., 1987; Kepecs et al., 2007; Verhagen et al., 2007). Similarly, they scan nearby objects by rhythmic movement of their mystacial whiskers, generated by contraction of muscle groups in the mystacial pad, referred to as whisking (Welker, 1964; Carvell and Simons, 1990; Hartmann, 2001; Berg and Kleinfeld, 2003; Bosman et al., 2011;

\footnotetext{
Received Aug. 13, 2012; revised March 12, 2013; accepted April 2, 2013.

Author contributions: S.R., B.H., and A.K. designed research; S.R. and A.K. performed research; B.H. contributed unpublished reagents/analytic tools; S.R., B.H., and A.K. analyzed data; S.R., B.H., and A.K. wrote the paper.

*S.R. and B.H. contributed equally to this work.

This research was supported by grants from the National Institutes of Health National Institute of Neurological Disorders and Stroke and Klingenstein and Sloan Foundations. B.H. received support from the Swartz Foundation and Marie Curie International Outgoing Fellowship within the EU Seventh Framework Programme for Research and Technological Development. A.K. is grateful for support from Zachary Mainen, in whose laboratory this project was initiated. We thank David Kleinfeld for discussions and sharing unpublished results, Florin Albeanu for comments on this manuscript, and Barry Burbach for technical assistance.

Correspondence should be addressed to Adam Kepecs, Cold Spring Harbor Laboratory, 1 Bungtown Road, Cold Spring Harbor, NY 11724. E-mail: kepecs@cshl.edu.

DOI:10.1523/JNEUROSCI.3874-12.2013

Copyright $\odot 2013$ the authors $\quad 0270-6474 / 13 / 338250-07 \$ 15.00 / 0$
}

Deschênes et al., 2012). Welker (1964) showed in his classic study using video analysis that exploratory sniffing and whisking are part of a coordinated motor sequence also involving head and nose movements that repeat with a frequency falling in the theta range $(4-12 \mathrm{~Hz})$. Here we revisit this observation and quantitatively evaluate whether sniffing and whisking are indeed synchronized on a cycle-by-cycle basis.

The temporal coordination of rhythmic sensorimotor behaviors may be important for several reasons. First, perceptual decisions relying on multimodal information may require the temporal alignment of sensory streams (Burr and Alais, 2006). Sniffing and whisking strongly pattern the flow of sensory information into the CNS and the firing of many olfactory and somatosensory neurons is locked to these rhythms (Adrian, 1950; Ahissar et al., 1997; Fee et al., 1997; Kleinfeld et al., 2002; Cang and Isaacson, 2003; Buonviso et al., 2006; Crochet and Petersen, 2006; Leiser and Moxon, 2007; Khatri et al., 2009; Cury and Uchida, 2010; Dhawale et al., 2010; Shusterman et al., 2011; Schaefer and Margrie, 2012); therefore, their phase locking would facilitate cross-modal linking of the perception of smell and touch. In fact a recently discovered snout muscle group may be specialized to coordinate nostril positioning with sniffing and whisking (Haidarliu et al., 2012).

Second, such sensorimotor synchrony may be a reflection of a broader coordination of processing between multiple brain regions. For instance, visual and auditory cortices can be rhythmically synchronized during attentional selection (Lakatos et al., 2008). In addition, attention can sample the environment in a rhythmic fashion at theta frequencies even in the absence of ex- 
plicit sensory patterning (Landau and Fries, 2012). Finally, the coordination of rhythmic sensorimotor behaviors could be also advantageous from a bioenergetics standpoint. For instance, a classic exploration of the relationship between breathing and locomotion (Alexander, 1993; Bramble and Jenkins, 1993) or wing beats (Berger et al., 1970) revealed complex patterns of rhythmic coordination critical for efficient respiration.

Therefore, to probe the interdependencies in active sensing processes during exploration, we simultaneously recorded breathing and whisking in freely moving rats foraging for food pellets and statistically evaluated their cycle-by-cycle coordination. We found that the two sensorimotor processes were strongly phase coupled even when the fundamental frequencies of their rhythms were different.

\section{Materials and Methods}

All procedures involving animals were performed in accordance with National Institutes of Health standards as approved by the Cold Spring Harbor Laboratory Institutional Animal Care and Use Committee.

Auditory cued pellet chasing task. Male Long-Evans hooded rats $(n=4)$ were placed in a large circular arena (diameter, $75 \mathrm{~cm})$ and conditioned on an auditory cued pellet-chasing task to search for food pellets. An auditory cue was played on a random interval schedule every 30-45 s. After a delay of $4 \mathrm{~s}$ from the onset of the auditory cue (except one session with $7 \mathrm{~s}$ ), a single $20 \mathrm{mg}$ bacon-flavored pellet (BioServ) was dropped into the arena from an overhead pellet dispenser (ENV-203; Med Associates). Rats searched for the pellets by actively exploring the arena.

Position tracking. The position of the rat in the arena was monitored at $30 \mathrm{~Hz}$ by tracking one red and one green LED integrated into the preamplifier on the microdrive (Neuralynx). $X$ and $Y$ coordinates were lowpass filtered at $4 \mathrm{~Hz}$ to remove high-frequency fluctuations and the instantaneous speed was calculated. The spatial resolution of the tracking was $1.5 \mathrm{~mm} /$ pixel.

Measurement of respiration. Rats were implanted with a temperature sensor (0.005 in. Teflon-coated thermocouple, Omega, part \#5TC-TTK-36-36) in one nostril through a hole made in the skull $\sim 7.7 \mathrm{~mm}$ anterior to the cribriform suture and secured with dental acrylic (see Fig. $1 A$ ) as described previously (Kepecs et al., 2007). Thermocouple measures the cooling and warming of air as it passes through the nasal cavity. Although junction potentials make it difficult to interpret the absolute voltage recorded, the timing of inhalations and exhalations can be readily determined from the cooling-warming patterns. Direct measurements of chest expansion using a piezoelectric belt wrapped around the rat's body in pilot experiments revealed that thermocouple and plethysmograph signals agreed on a cycle-by-cycle basis (Kepecs et al., 2007). Thermocouple signals were acquired at $1000 \mathrm{~Hz}$, amplified, and filtered between 0.1 and $475 \mathrm{~Hz}$.

Measurement of the mystacial electromyogram. Differential mystacial electromyogram (EMG) was recorded bilaterally from the intrinsic muscles using techniques previously described (Carvell et al., 1991; Berg and Kleinfeld, 2003). Briefly, microwire EMG electrodes made of Tefloncoated $50 \mu \mathrm{m}$ tungsten wire (A-M Systems) were surgically implanted in the mystacial pad to record the activity of intrinsic muscles (Fig. 1 A,B). A midline incision was made in the skin and a 25 gauge syringe needle threaded with four EMG electrodes stripped at the tip was inserted through the muscular tissue of the mystacial pad, exiting the skin from the rostroventral side. The needle was pulled back, leaving the electrodes in the mystacial pad. The electrodes were adjusted to record different portions of the pad. The EMG was digitized at $1000 \mathrm{~Hz}$. To confirm positioning of EMG electrodes, biphasic stimulation pulses were delivered at $150-200 \mu \mathrm{A}$ under anesthesia, which elicited robust protraction of the whisker pad (Berg and Kleinfeld, 2003).

Classification of trials by sniffing frequency in the pre-pellet period. Sniffing segments around the timing of each pellet drop were extracted $(8 \mathrm{~s}$ long), detrended by subtracting the best-fit line, $z$-score normalized, and low-pass filtered at $100 \mathrm{~Hz}$. Since sniffing frequency during the pre-pellet drop period was variable (mostly depending on whether a rat was engaged in active exploration), we used a median split to define two sniffing frequency bands: low $(2-6.5 \mathrm{~Hz})$ and high $(6.5-11.5 \mathrm{~Hz})$ frequencies. The difference-to-sum ratio for peak power in these two frequency bands during the $8 \mathrm{~s}$ pre-pellet drop period was calculated yielding a value between -1 and 1 . Values closer to 1 correspond to trials in which there is more power predominantly in the lower frequency band and vice versa. An absolute threshold $>0.5$ was selected to classify trials into two groups: low sniffing and high sniffing trials.

Spectrogram and coherogram. Pellet drop aligned segments (8 s perievent) of raw whisker EMG were detrended, $z$-score normalized, highpass filtered at $20 \mathrm{~Hz}$, and the timing of peaks with amplitudes $>2$ SDs were included in the whisking multi-unit point process. Trial-averaged spectrograms for sniffing and whisking multi-unit as well as coherograms between sniffing and whisking multi-unit were calculated using multitaper spectral methods implemented in the Chronux package (www.chronux.org) (Mitra and Bokil, 2007).

Coherence. For each animal, five segments of high signal-to-noise ratio (SNR) of the whisking EMG were randomly selected to provide a balanced sample across animals despite the difference in the number of sessions and recording time. This selection procedure did not have a substantial influence on the shape of the coherence functions compared with coherence calculated for all recorded data. Linear trends were removed from the respiration signal and the root-mean-square (RMS) of the EMG by subtracting the best line fit from the signals. Magnitude squared coherence was calculated by using Welch's periodogram method with a Hanning window function and $50 \%$ overlapping windows.

Determination of coupling mode between sniffing and whisking. The timing of each whisking bout was determined in the following manner. EMG signals were high-pass filtered at $10 \mathrm{~Hz}$ to remove slow trends and the RMS was computed using 10 or 20 ms nonoverlapping windows. The RMS was high-pass filtered at $50 \mathrm{~Hz}$ and rectified to completely remove baseline fluctuations. Individual whisking bouts were detected by localizing RMS peaks passing a user-defined threshold customized for each session to ensure inclusion of all whisks while avoiding baseline noise. A point process of whisks was generated by taking the time points when the raw EMG reached its maximum within each whisking bout. This peak detection algorithm was ideally suited for assigning the different sniffing-whisking modes because it enabled us to count the number of whisks per sniff cycle. Different types of phase locking such as 2:1, 1:1, and 1:2 (referred to as "modes of phase locking") were determined based on the ratio of whisks and sniffing cycles. Epochs were classified to different phase-coupling modes only if they were longer than half a second and contained at least four whisks. Frequency relationship of the two oscillators in the 1:2 and 2:1 modes was tested using a bootstrap test (resampled 1000 times) to assess whether the higher frequency was significantly different from the second harmonic (double) of the lower frequency. To get independent samples for sniffing and whisking frequency, the reciprocal of the mean period length (time differences between inhalation start points or whisking bouts) was used for frequency estimation in this analysis. No significant difference between sniffing frequency and the second harmonic of whisking frequency in the 1:2 mode was detected (7.595 Hz vs $7.591 \mathrm{~Hz}, p=0.95)$. Likewise, whisking frequency was not significantly different from the second harmonic of the sniffing frequency in the $2: 1$ mode $(6.822 \mathrm{~Hz}$ vs $6.723 \mathrm{~Hz}, p=0.58)$.

Preferred whisking phase. The sniffing signal was low-pass filtered at $30 \mathrm{~Hz}$ to enhance SNR by removing high-frequency components. Hilbert-transform of the filtered and $z$-scored signal was calculated and respiration phase was determined by taking the angle of the complex analytic signal (Fig. 1C). The major advantage of this algorithm is that it estimates the phase of any quasi-periodic signal in a temporally refined manner without interpolation, defining a time series that continuously reflects the "instantaneous phase" of the ongoing oscillation (Hurtado et al., 2004). To quantify the precise phase relationship of whisking and sniffing, the whisking RMS was used, as it provides continuous information on whisking activity in the time domain, as opposed to the temporally restricted, discrete information of the point process. Mean whisking as a function of sniffing phase 

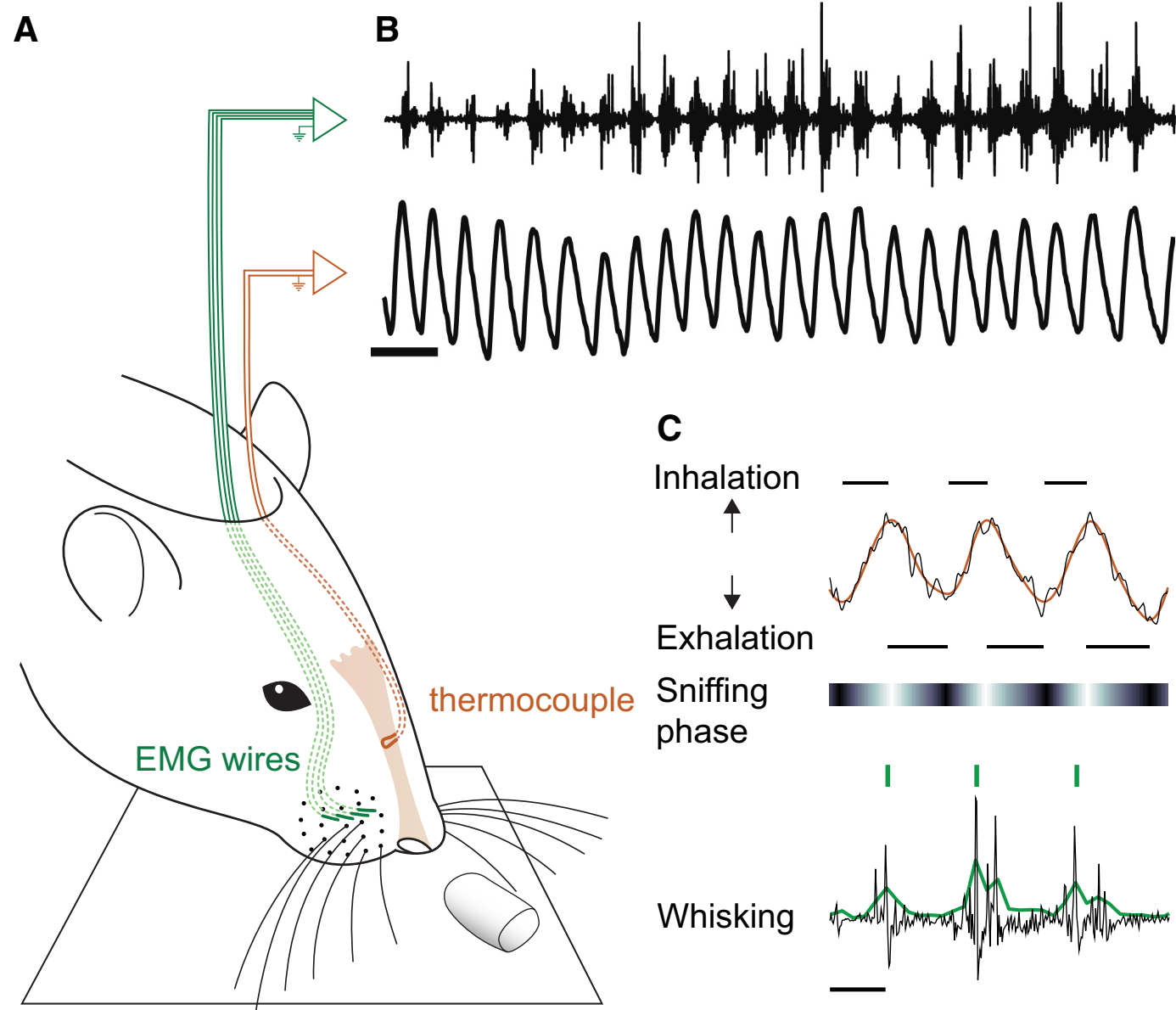

Figure 1. Simultaneous recording of sniffing and whisking in awake freely moving rats. $\boldsymbol{A}$, Schematic of recording. Breathing was monitored using a thermocouple and concurrent EMG was recorded from the mystacial pad. $\boldsymbol{B}$, Example of raw traces of simultaneously recorded sniffing and whisking. Scale bar, $200 \mathrm{~ms}$. $\boldsymbol{C}$, The raw breathing signal (top, black trace) was processed through a low-pass filter ( $30 \mathrm{~Hz}$; orange, filtered trace) and respiration phase was calculated by Hilbert transform (see Materials and Methods; horizontal bar shows respiration phase; white, zero phase). The raw EMG (bottom, black trace) was high-pass filtered, then squared and smoothed by an RMS algorithm (green trace) and whisking bouts were detected through a thresholding procedure for automatic detection of different modes of phase coupling (see Materials and Methods; green bars). Scale bar, 50 ms.

was calculated by pooling all sessions for each rat and averaging whisking RMS over rats in 60 degree bins with 50 degrees of overlap.

Relation of coupling mode to behavioral events. The relative prevalence of the 2:1, 1:1, and 1:2 modes $2-8 \mathrm{~s}$ before and after pellet drops was determined by counting the epochs that overlapped with the behavioral windows. The window boundaries were determined based on the change in coherence after pellet drops (see Fig. $2 F$ ). Relative frequencies were compared for inside and outside these windows as well as for windows before and after pellet drops. Statistical evaluation was performed by using $\chi^{2}$ test $(p<0.05)$.

\section{Results}

\section{Sniffing and whisking are coherent during exploration}

We set out to investigate whether two prominent sensorimotor rhythms, sniffing and whisking, occur in a temporally coordinated fashion during active exploration. To this end, we simultaneously recorded breathing by measuring temperature changes in the nasal cavity with an implanted thermocouple and whisking by measuring the electromyogram of the intrinsic muscles in the mystacial pad (Fig. 1). During recording, rats were engaged in a pellet-chasing task, foraging in a circular arena for food pellets (Fig. 2A). An auditory cue predicted the availability of food pellet after a fixed delay. Food pellets were dropped from an overhead dispenser at random locations within the arena, prompting rats to engage in frequent bouts of exploration to discover their location. There was a robust increase in speed of the rat after a pellet drop (speed before pellet drop, $15.8 \pm 0.6 \mathrm{~cm} / \mathrm{s}$; speed after pellet drop, $18.5 \pm 0.6 \mathrm{~cm} / \mathrm{s}$, mean \pm SEM, two-tailed paired $t$ test; $p=$ $\left.1.4 \times 10^{-7}\right)$. Visual observation suggested that rats were performing the classic exploratory movement sequence described by Welker (1964) involving rhythmic head movements, sniffing, and whisking as they explored the arena.

Examination of the concurrently recorded thermocouple and EMG signals revealed that sniffing and whisking emerged in a strongly rhythmic fashion (Fig. $2 B$ ). The frequency of these rhythms changed dynamically within the theta frequency range $(4-12 \mathrm{~Hz})$, as reported previously (Youngentob et al., 1987; Gao et al., 2001; Berg and Kleinfeld, 2003; Uchida and Mainen, 2003; Kepecs et al., 2007; Verhagen et al., 2007; Deschênes et al., 2012). The behavior of rats was variable before the pellet was delivered, both in terms of speed as well as sniffing and whisking frequency. Ejection of the food pellet initiated robust exploratory behavior that was associated with higher frequency sniffing and whisking (Fig. 2B). Therefore we classified all trials into two groups based on the pre-pellet drop sniffing frequency (Fig. 2B; see Materials and Methods) and found that the average speed in the pre-pellet drop period was significantly greater for high compared with low sniffing frequency trials $\left(10.8 \pm 1.0\right.$ vs $16.4 \pm 0.8 \mathrm{~cm} / \mathrm{s} ; p=3.8 \times 10^{-5}$ two-tailed $t$ test). We computed the average time-frequency 
A

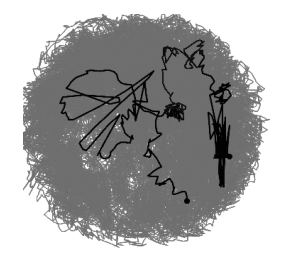

C

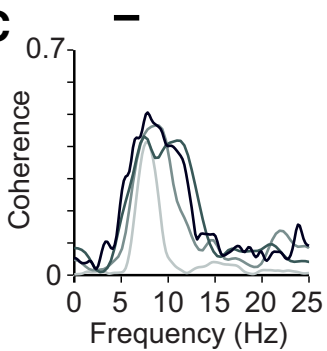

D
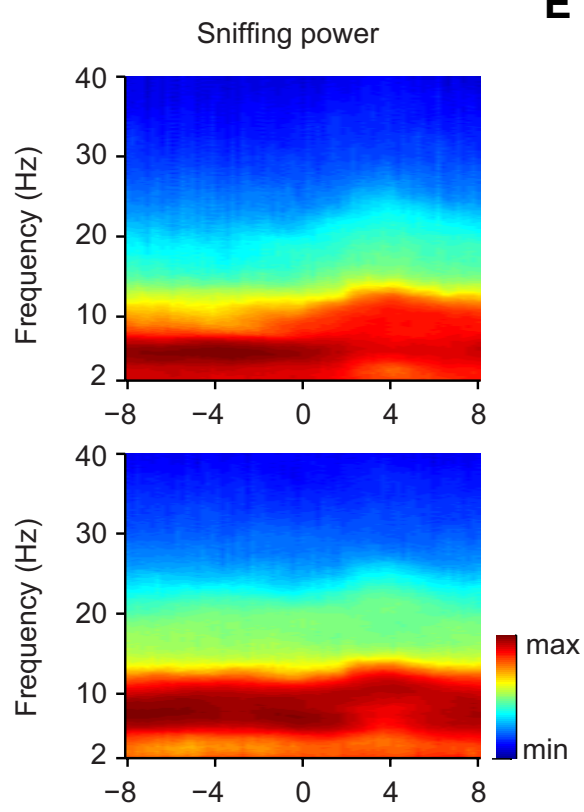

E
B Cue onset

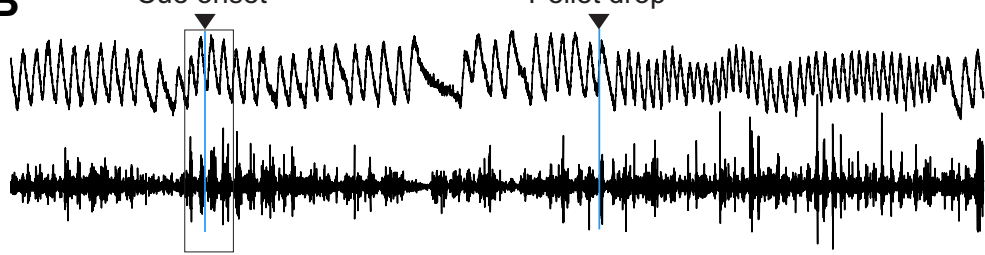

Pellet drop
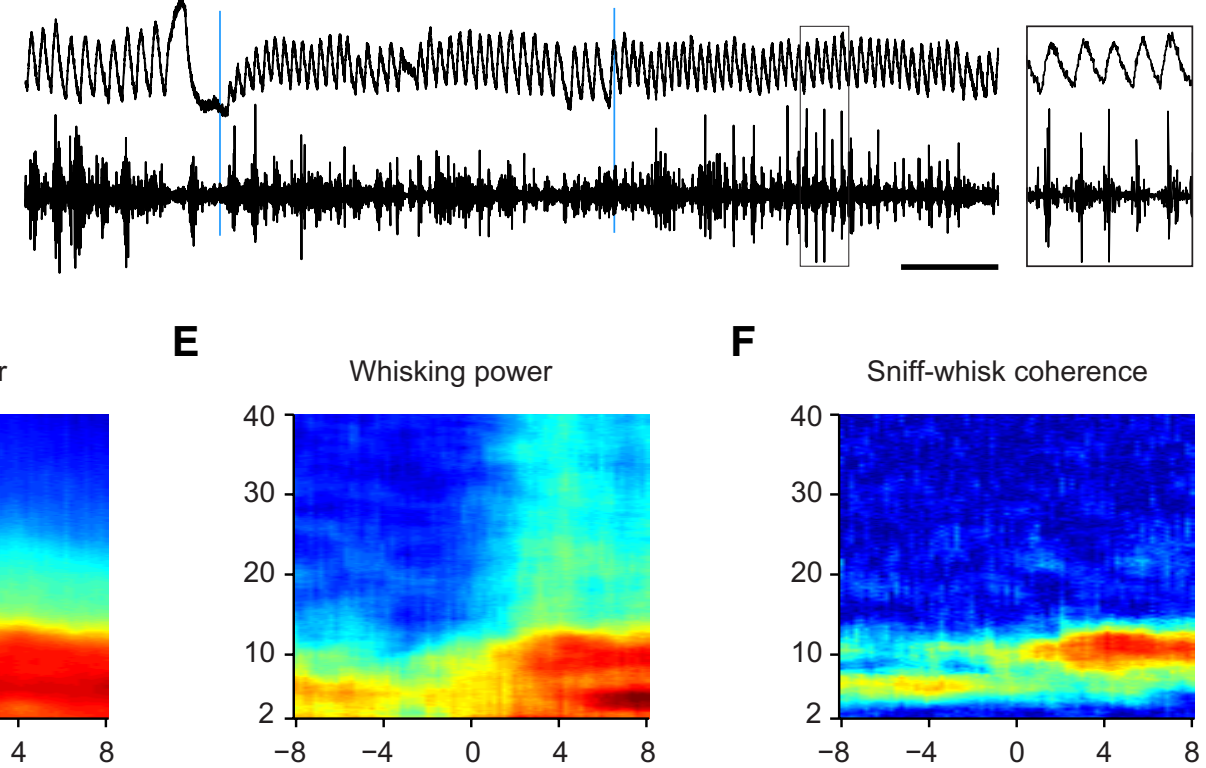

$\mathbf{F}$

Sniff-whisk coherence

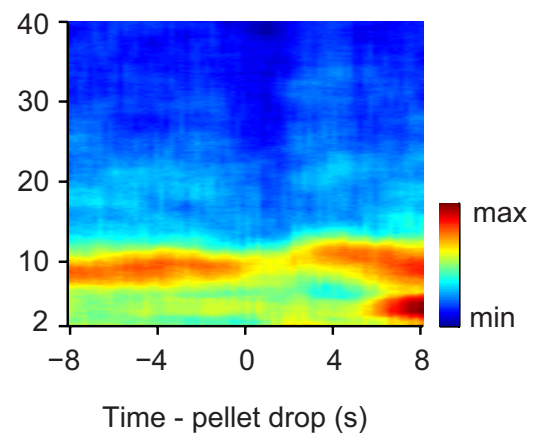

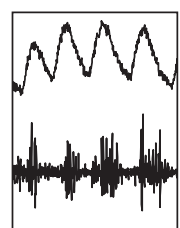
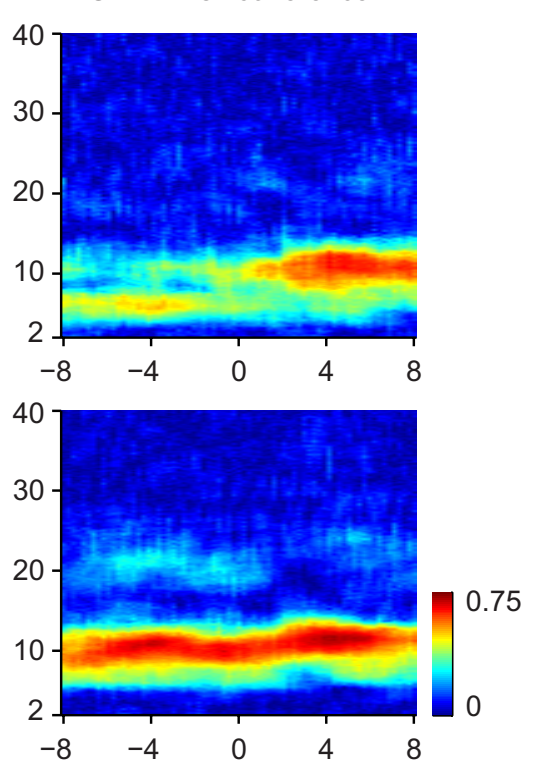

Figure 2. Sniffing and whisking are coherent at theta frequencies. $A$, Path traversed by the rat on a single cued trial while searching for a food pellet. Gray lines show the path during the entire session. Scale bar, $7.5 \mathrm{~cm}$. B , Two examples of concurrently recorded sniffing and whisking traces during two trials of the pellet chasing task. At 4 s from cue onset, the pellet dropped into the arena from the dispenser. Note the increase in sniffing as well as whisking frequency after the pellet drop during active search. $\boldsymbol{C}$, Average sniffing-whisking coherence across sniff epochs shown for all four rats. $\boldsymbol{D}-\boldsymbol{F}$, Trial-averaged spectrogram of sniffing $(\boldsymbol{D})$, whisking $(\boldsymbol{E})$, and coherence between sniffing and whisking $(\boldsymbol{F})$ aligned to pellet drop. Trials were sorted according to low (upper row) and high (lower row) pre-pellet sniffing frequency (see Materials and Methods).

spectrograms of the respiration and EMG signals aligned to the pellet drop. Figure 2, $D$ and $E$, show that the frequencies of the two rhythms broadly matched during different behavioral epochs including anticipation (before pellet drop) and active search (after pellet drop). However, two oscillations with similar frequencies are not necessarily temporally coordinated (Berg et al., 2006). Therefore to test whether the two rhythms are interrelated, we computed spectral coherence between respiration and whisker muscle activity. Figure $2 F$ shows that sniffing and whisking are strongly coherent across different epochs in both low- and high-frequency sniffing trials. We further examined this correlation during sniffing bouts selected based on the presence of whisking activity, regardless of foraging behavior. Across these bouts, we found strong coherence between sniffing and whisking in the theta range in all four rats (Fig. 2C). These analyses confirm the long-held hypothesis that active, rhythmic olfactory and somatosen- sory sampling are temporally coordinated (Welker, 1964; Semba and Komisaruk, 1984).

\section{Multiple modes of phase locking between sniffing and whisking}

Coherence between sniffing and whisking informs us about the temporal coordination of the two signals, but does not provide insights into possible modes of coupling that could result in a similar coherence spectrum. To reveal the fine structure of sniffing-whisking coordination, we analyzed the phase relationship of unitary whisking bouts relative to the respiration cycle. We found that whisking activity occurred at similar phases of sniffing across different cycles of respiration, resulting in a strong phase locking between the two rhythms (Fig. 3). Specifically, whisker protraction was aligned with the inhalation phase of sniffing (Fig. 3B). 
We next investigated how phase locking between sniffing and whisking might change on a cycle-by-cycle basis. By quantifying the numbers of whisks within consecutive cycles of sniffing, we uncovered multiple modes of sniffingwhisking phase coupling. The most prevalent mode of locking during exploration showed one-to-one coupling between whisks and sniffs, that is, each inhalation was accompanied by a single whisker protraction (Fig. 3B; 1:1 mode). In addition, we also observed other modes of coupling. In one mode, whisks occurred in only every other sniff cycle (Fig. 3C, 1:2 mode), whereas in another, two whisking episodes were present in every sniff cycle (Fig. 3A, 2:1 mode). Thus, although sniffing and whisking showed different fundamental frequencies in these modes of coupling, they were still phase locked over multiple cycles. The precise relationship between sniffing and whisking is illustrated as a raster representation (Fig. $3 A-C$, bottom) for $2: 1,1: 1$, and $1: 2$ whisk-sniff locking, the most prevalent modes in our dataset. These sniffing-whisking raster plots reveal that the precise timing of whisks relative to the respiratory cycle is maintained for all locking modes over a range of fundamental frequencies (Fig. 3A-C, bottom). Interestingly, across all these modes one rhythm stays within the theta frequency range while the other is at its half frequency (Fig. $3 D)$, so that the higher frequency rhythm can be thought of as a second harmonic of the lower, fundamental frequency (see Materials and Methods).

Next we quantified whether these different modes also involve changes in the relative timing of the two rhythms. We computed the average whisking activity as a function of the sniffing phase. We found that all observed modes of sniffing-whisking coupling showed the same preferred phase (Fig. $3 E$ ), demonstrating that inhalation and whisker protraction are phase aligned.

Different modes of phase locking might be related to specific behavioral contingencies. To assess this we analyzed the prevalence of these modes around the pellet drop. We found that while 1:1 locking dominated our recordings and all modes could occur either close to or distant from behavioral events, the 1 whisk to 2 sniffs mode was significantly clustered after pellet drops (18.3\% of all 1:2 epochs) as compared with both the $1: 1\left(14.4 \%\right.$ of all $1: 1$ epochs, $p=0.034, \chi^{2}$ test $)$ and the $2: 1$ modes $\left(9.0 \%\right.$ of all $2: 1$ epochs, $p=0.009, \chi^{2}$ test). Moreover, in low pre-pellet sniffing frequency trials, $1: 1$ and 1:2 modes were significantly more prevalent during the period after compared to before pellet drop $(p=0.040$ and $p=0.012$, respectively, $\chi^{2}$ test).

The 1:2 mode was also accompanied by significantly greater animal speed $(18.8 \pm 0.70 \mathrm{~cm} / \mathrm{s}$, mean $\pm \mathrm{SEM})$ compared with
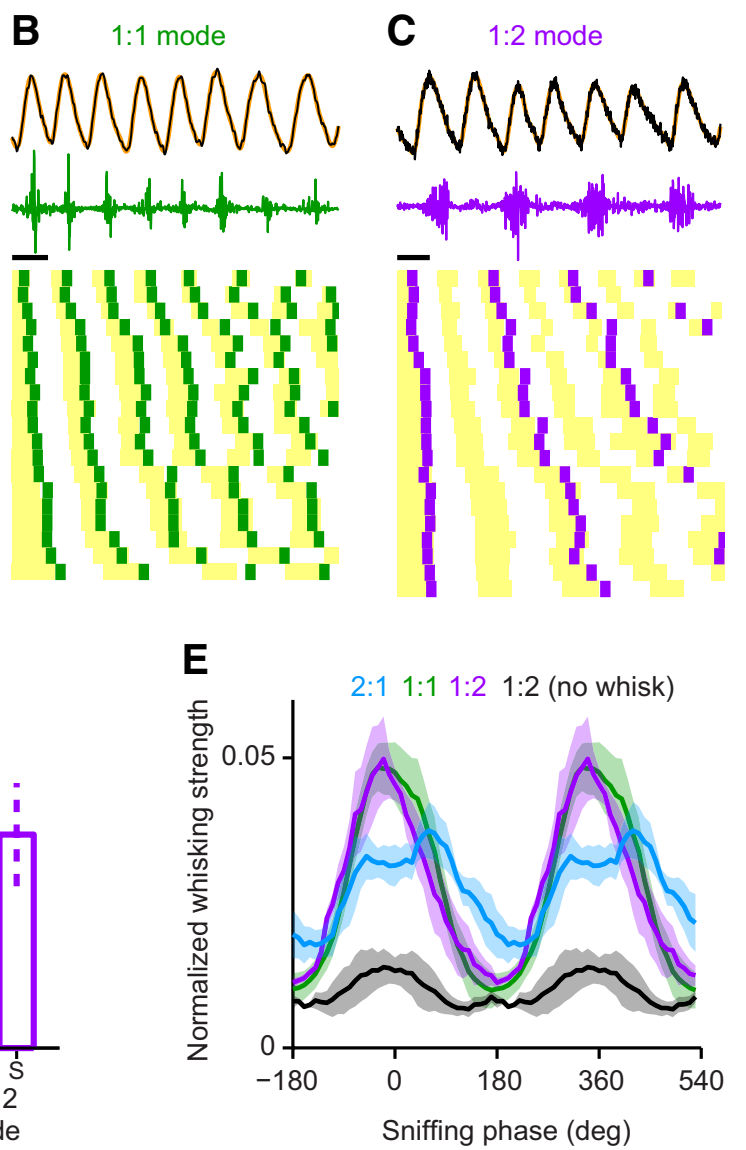

Figure 3. Distinct phase-locking modes of whisking relative to sniffing. $\boldsymbol{A}-\boldsymbol{C}$, Top, example traces of sniffing and corresponding whisking. Bottom, raster representation of additional examples with different sniffing frequencies, sorted 作 iff cycle is accompanied by a single whisking bout. $C$, One-to-two locking, whisking bouts occur in every second sniffing coupling. Dashed error bars indicate SD. E, Average phase preference of whisking relative to sniffing. The RMS of whisking was averaged over overlapping bins of sniffing phase to provide a phase-dependent estimate of whisking strength (see d Blue, two-to-one; green, one-to-one; purple, one-to-two coupling; black, alternating cycles in -to-two coupling that lack a whisking bout reaching detection threshold show very weak phase locking. Shading indicates SEM across animals. Note that whisking phase was more reliable in the 1:1 and 1:2 modes than in the 2:1 mode.

the $1: 1$ mode $\left(14.83 \pm 0.24 \mathrm{~cm} / \mathrm{s}, p=2.6 \times 10^{-9}\right.$, MannWhitney test) and $2: 1$ modes $(13.14 \pm 0.98 \mathrm{~cm} / \mathrm{s}, p=1.4 \times$ $10^{-5}$, Mann-Whitney test). These findings suggest that the coupling mode between sniffing and whisking might be dynamically controlled in coordination with the behavior.

\section{Discussion}

It has long been observed that during exploration of their environment, rats simultaneously sample olfactory and tactile stimuli with rapid respiratory movements, sniffing, and concurrent brisk motion of their vibrissae, whisking (Welker, 1964). Here, we simultaneously monitored and quantified breathing and whisking in rats exploring an arena for food reward. We observed that these two sensorimotor rhythms were coherent in the theta frequency range and showed strong phase locking during exploration. Moreover, apart from the predominant cycle-by-cycle, 1:1 phase coupling, we also observed multiple modes of locking between breathing and whisking occurring at different fundamental frequencies.

A recent study measured sniffing and whisking in mice and did not observe phase locking between the two rhythms except at low frequencies corresponding to breathing (Cao et al., 
2012). This discrepancy with our data may be attributed to the use of an external thermocouple to measure sniffing and video monitoring of single vibrissa to record whisking, methods that may be less precise in capturing the relative timing of the two processes. Nevertheless, our data do not rule out the possibility that in some behavioral states sniffing and whisking are not phase locked.

Most rhythmic motor patterns are produced by central pattern generators (CPGs) in the brainstem (Grillner, 2006). Brainstem nuclei, including the pre-Botzinger and Botzinger complexes, have been implicated in generating both sniffing and whisking rhythms (Smith et al., 1991; Hattox et al., 2003; Tan et al., 2008); however, it is not known whether the breathing CPG is also involved in generating whisking (Gao et al., 2001; Hattox et al., 2002). Because sniffing and whisking occur in a relatively broad frequency band (Fig. 2), it is particularly interesting that we observed strong one-to-one phase locking at different frequencies within the theta range and multimode locking when the two rhythms were oscillating at different fundamental frequencies. This suggests that these two rhythms are under the control of two interdependent, dynamically coordinated CPGs. It will be interesting to investigate whether there are more complex modes of coupling, such as phase precession, how mode transitions occur, and the neural mechanisms responsible for coordination (Deschênes et al., 2012).

Although sniffing and whisking showed phase locking at multiple modes, the preferred phase of coordination of these two rhythms remained unchanged. Whisking protraction was always coupled to the inspiratory phase of sniffing, suggesting that the stimulus acquisition phases of the two rhythms are coordinated. This provides support for the long held idea that during exploration rats coordinate sensory acquisition across multiple modalities (Welker, 1964). Previous studies in rodents have shown that each sniff is a "snapshot" of the olfactory world (Uchida and Mainen, 2003; Kepecs et al., 2006). Our results suggest that in fact rats may acquire a more general multisensory snapshot of their surroundings.

Sniffing and whisking strongly influence the neural coding of sensory information across olfactory (Adrian, 1950; Cang and Isaacson, 2003; Buonviso et al., 2006; Cury and Uchida, 2010; Dhawale et al., 2010; Shusterman et al., 2011; Schaefer and Margrie, 2012) and somatosensory areas (Ahissar et al., 1997; Fee et al., 1997; Kleinfeld et al., 2002; Crochet and Petersen, 2006; Leiser and Moxon, 2007). Many neurons outside the olfactory system such as the locus ceruleus (Cohen and Wang, 1959), amygdala (Frysinger et al., 1988), and hippocampus (Macrides et al., 1982) can lock their firing to sniffing. On the other hand, the conditions under which whisking synchronizes with the hippocampal theta rhythm are not well understood (Komisaruk, 1970; Berg et al., 2006). Theta oscillations have been observed in a range of brain areas (Buzsáki, 2002) and there have been reports of dynamic theta phase coupling between the hippocampus and medial prefrontal cortex (Hyman et al., 2005; Siapas et al., 2005; Young and McNaughton, 2009) and amygdala (Paré and Gaudreau, 1996; Seidenbecher et al., 2003), as well as the striatum (Berke et al., 2004; van der Meer and Redish, 2011). These observations have lead to the suggestion that under some behavioral contingencies the theta cycle could provide a common protocol for coordinating neural processing across brain areas (Komisaruk, 1977; Hasselmo, 2005; Buzsáki, 2006; Kepecs et al., 2006). Supporting this notion, our results suggest that somatosensory and olfactory information processing are phase coordinated at theta frequencies.

\section{References}

Adrian ED (1950) The electrical activity of the mammalian olfactory bulb. Electroencephalogr Clin Neurophysiol 2:377-388. CrossRef Medline

Ahissar E, Haidarliu S, Zacksenhouse M (1997) Decoding temporally encoded sensory input by cortical oscillations and thalamic phase comparators. Proc Natl Acad Sci U S A 94:11633-11638. CrossRef Medline

Alexander RM (1993) Breathing while trotting. Science 262:196-197. CrossRef Medline

Atema J (1995) Chemical signals in the marine environment: dispersal, detection, and temporal signal analysis. Proc Natl Acad Sci U S A 92:62-66. CrossRef Medline

Berg RW, Kleinfeld D (2003) Rhythmic whisking by rat: retraction as well as protraction of the vibrissae is under active muscular control. J Neurophysiol 89:104-117. Medline

Berg RW, Whitmer D, Kleinfeld D (2006) Exploratory whisking by rat is not phase locked to the hippocampal theta rhythm. J Neurosci 26:6518-6522. CrossRef Medline

Berger M, Roy OZ, Hart JS (1970) The co-ordination between respiration and wing beats in birds. J Comp Physiol 66:190-200.

Berke JD, Okatan M, Skurski J, Eichenbaum HB (2004) Oscillatory entrainment of striatal neurons in freely moving rats. Neuron 43:883-896. CrossRef Medline

Bosman LW, Houweling AR, Owens CB, Tanke N, Shevchouk OT, Rahmati N, Teunissen WH, Ju C, Gong W, Koekkoek SK, De Zeeuw CI (2011) Anatomical pathways involved in generating and sensing rhythmic whisker movements. Front Integr Neurosci 5:53. Medline

Bramble DM, Jenkins FA Jr (1993) Mammalian locomotor-respiratory integration: implications for diaphragmatic and pulmonary design. Science 262:235-240. CrossRef Medline

Buonviso N, Amat C, Litaudon P (2006) Respiratory modulation of olfactory neurons in the rodent brain. Chem Senses 31:145-154. Medline

Burr D, Alais D (2006) Combining visual and auditory information. Prog Brain Res 155:243-258. CrossRef Medline

Buzsáki G (2002) Theta oscillations in the hippocampus. Neuron 33:325-340. CrossRef Medline

Buzsáki G (2006) Rhythms of the brain. New York: Oxford UP.

Cang J, Isaacson JS (2003) In vivo whole-cell recording of odor-evoked synaptic transmission in the rat olfactory bulb. J Neurosci 23:4108-4116. Medline

Cao Y, Roy S, Sachdev RN, Heck DH (2012) Dynamic correlation between whisking and breathing rhythms in mice. J Neurosci 32:1653-1659. CrossRef Medline

Carvell GE, Simons DJ (1990) Biometric analyses of vibrissal tactile discrimination in the rat. J Neurosci 10:2638-2648. Medline

Carvell GE, Simons DJ, Lichtenstein SH, Bryant P (1991) Electromyographic activity of mystacial pad musculature during whisking behavior in the rat. Somatosens Mot Res 8:159-164. CrossRef Medline

Cohen MI, Wang SC (1959) Respiratory neuronal activity in pons of cat. J Neurophysiol 22:33-50. Medline

Crochet S, Petersen CC (2006) Correlating whisker behavior with membrane potential in barrel cortex of awake mice. Nat Neurosci 9:608-610. CrossRef Medline

Cury KM, Uchida N (2010) Robust odor coding via inhalation-coupled transient activity in the mammalian olfactory bulb. Neuron 68:570-585. CrossRef Medline

Deschênes M, Moore J, Kleinfeld D (2012) Sniffing and whisking in rodents. Curr Opin Neurobiol 22:243-250. CrossRef Medline

Dhawale AK, Hagiwara A, Bhalla US, Murthy VN, Albeanu DF (2010) Nonredundant odor coding by sister mitral cells revealed by light addressable glomeruli in the mouse. Nat Neurosci 13:1404-1412. CrossRef Medline

Fee MS, Mitra PP, Kleinfeld D (1997) Central versus peripheral determinants of patterned spike activity in rat vibrissa cortex during whisking. J Neurophysiol 78:1144-1149. Medline

Frysinger RC, Zhang JX, Harper RM (1988) Cardiovascular and respiratory relationships with neuronal discharge in the central nucleus of the amygdala during sleep-waking states. Sleep 11:317-332. Medline

Gao P, Bermejo R, Zeigler HP (2001) Whisker deafferentation and rodent whisking patterns: behavioral evidence for a central pattern generator. J Neurosci 21:5374-5380. Medline 
Gomez-Marin A, Stephens GJ, Louis M (2011) Active sampling and decision making in Drosophila chemotaxis. Nat Commun 2:441. CrossRef Medline

Grillner S (2006) Biological pattern generation: the cellular and computational logic of networks in motion. Neuron 52:751-766. CrossRef Medline

Haidarliu S, Golomb D, Kleinfeld D, Ahissar E (2012) Dorsorostral snout muscles in the rat subserve coordinated movement for whisking and sniffing. Anat Rec 295:1181-1191. CrossRef Medline

Hartmann MJ (2001) Active sensing capabilities of the rat whisker system. Autonomous Robots 11:249-254. CrossRef

Hasselmo ME (2005) What is the function of hippocampal theta rhythm?Linking behavioral data to phasic properties of field potential and unit recording data. Hippocampus 15:936-949. CrossRef Medline

Hattox AM, Priest CA, Keller A (2002) Functional circuitry involved in the regulation of whisker movements. J Comp Neurol 442:266-276. CrossRef Medline

Hattox A, Li Y, Keller A (2003) Serotonin regulates rhythmic whisking. Neuron 39:343-352. CrossRef Medline

Hurtado JM, Rubchinsky LL, Sigvardt KA (2004) Statistical method for detection of phase-locking episodes in neural oscillations. J Neurophysiol 91:1883-1898. CrossRef Medline

Hyman JM, Zilli EA, Paley AM, Hasselmo ME (2005) Medial prefrontal cortex cells show dynamic modulation with the hippocampal theta rhythm dependent on behavior. Hippocampus 15:739-749. CrossRef Medline

Kepecs A, Uchida N, Mainen ZF (2006) The sniff as a unit of olfactory processing. Chem Senses 31:167-179. Medline

Kepecs A, Uchida N, Mainen ZF (2007) Rapid and precise control of sniffing during olfactory discrimination in rats. J Neurophysiol 98:205-213. CrossRef Medline

Khatri V, Bermejo R, Brumberg JC, Keller A, Zeigler HP (2009) Whisking in air: encoding of kinematics by trigeminal ganglion neurons in awake rats. J Neurophysiol 101:1836-1846. CrossRef Medline

Kleinfeld D, Sachdev RN, Merchant LM, Jarvis MR, Ebner FF (2002) Adaptive filtering of vibrissa input in motor cortex of rat. Neuron 34:10211034. CrossRef Medline

Komisaruk BR (1970) Synchrony between limbic system theta activity and rhythmical behavior in rats. J Comp Physiol Psychol 70:482-492. CrossRef Medline

Komisaruk BR (1977) The role of rhythmical brain activity in sensorimotor integration. In: Progress in psychobiology and physiological psychology (Sprague JM, Epstein AN, eds), pp 55-90. New York: Academic.

Lakatos P, Karmos G, Mehta AD, Ulbert I, Schroeder CE (2008) Entrainment of neuronal oscillations as a mechanism of attentional selection. Science 320:110-113. CrossRef Medline

Landau AN, Fries P (2012) Attention samples stimuli rhythmically. Curr Biol 22:1000-1004. CrossRef Medline

Leiser SC, Moxon KA (2007) Responses of trigeminal ganglion neurons during natural whisking behaviors in the awake rat. Neuron 53:117-133. CrossRef Medline
Macrides F, Eichenbaum HB, Forbes WB (1982) Temporal relationship between sniffing and the limbic theta rhythm during odor discrimination reversal learning. J Neurosci 2:1705-1717. Medline

Mitra P, Bokil H (2007) Observed brain dynamics. New York: Oxford UP.

Otero-Millan J, Troncoso XG, Macknik SL, Serrano-Pedraza I, MartinezConde S (2008) Saccades and microsaccades during visual fixation, exploration, and search: foundations for a common saccadic generator. J Vis 8(14):21.1-21.18. CrossRef Medline

Paré D, Gaudreau H (1996) Projection cells and interneurons of the lateral and basolateral amygdala: distinct firing patterns and differential relation to theta and delta rhythms in conscious cats. J Neurosci 16:3334-3350. Medline

Schaefer AT, Margrie TW (2012) Psychophysical properties of odor processing can be quantitatively described by relative action potential latency patterns in mitral and tufted cells. Front Syst Neurosci 6:30. Medline

Seidenbecher T, Laxmi TR, Stork O, Pape HC (2003) Amygdalar and hippocampal theta rhythm synchronization during fear memory retrieval. Science 301:846-850. CrossRef Medline

Semba K, Komisaruk BR (1984) Neural substrates of two different rhythmical vibrissal movements in the rat. Neuroscience 12:761-774. CrossRef Medline

Shusterman R, Smear MC, Koulakov AA, Rinberg D (2011) Precise olfactory responses tile the sniff cycle. Nat Neurosci 14:1039-1044. CrossRef Medline

Siapas AG, Lubenov EV, Wilson MA (2005) Prefrontal phase locking to hippocampal theta oscillations. Neuron 46:141-151. CrossRef Medline

Smith JC, Ellenberger HH, Ballanyi K, Richter DW, Feldman JL (1991) PreBötzinger complex: a brainstem region that may generate respiratory rhythm in mammals. Science 254:726-729. CrossRef Medline

Tan W, Janczewski WA, Yang P, Shao XM, Callaway EM, Feldman JL (2008) Silencing preBötzinger Complex somatostatin-expressing neurons induces persistent apnea in awake rat. Nat Neurosci 11:538-540. CrossRef Medline

Uchida N, Mainen ZF (2003) Speed and accuracy of olfactory discrimination in the rat. Nat Neurosci 6:1224-1229. CrossRef Medline

van der Meer MA, Redish AD (2011) Theta phase precession in rat ventral striatum links place and reward information. J Neurosci 31:28432854. CrossRef Medline

Verhagen JV, Wesson DW, Netoff TI, White JA, Wachowiak M (2007) Sniffing controls an adaptive filter of sensory input to the olfactory bulb. Nat Neurosci 10:631-639. CrossRef Medline

Welker W (1964) Analysis of sniffing of the albino rat. Behaviour 22:223244. CrossRef

Young CK, McNaughton N (2009) Coupling of theta oscillations between anterior and posterior midline cortex and with the hippocampus in freely behaving rats. Cereb Cortex 19:24-40. Medline

Youngentob SL, Mozell MM, Sheehe PR, Hornung DE (1987) A quantitative analysis of sniffing strategies in rats performing odor detection tasks. Physiol Behav 41:59-69. CrossRef Medline 